\title{
Asymptotically polynomial solutions of difference equations
}

Janusz Migda*

\section{"Correspondence:}

migda@amu.edu.pl

Faculty of Mathematics and

Computer Science, A. Mickiewicz

University, Umultowska 87, Poznań,

61-614, Poland

\begin{abstract}
Asymptotic properties of solutions of a difference equation of the form

$\Delta^{m} x_{n}=a_{n} f\left(n, x_{\sigma(n)}\right)+b_{n}$

are studied. We present sufficient conditions under which, for any polynomial $\varphi(n)$ of degree at most $m-1$ and for any real $s \leq 0$, there exists a solution $x$ of the above equation such that $x_{n}=\varphi(n)+\mathbf{o}\left(n^{s}\right)$. We give also sufficient conditions under which, for given real $s \leq m-1$, all solutions $x$ of the equation satisfy the condition $x_{n}=\varphi(n)+o\left(n^{5}\right)$ for some polynomial $\varphi(n)$ of degree at most $m-1$.

MSC: $39 \mathrm{~A} 10$
\end{abstract}

Keywords: difference equation; asymptotically polynomial solution; prescribed asymptotic behavior

\section{Introduction}

Let $\mathbb{N}, \mathbb{Z}, \mathbb{R}$ denote the set of positive integers, the set of all integers and the set of real numbers, respectively. For $p, k \in \mathbb{Z}$, let $\mathbb{N}(p)=\{p, p+1, \ldots\}, \mathbb{N}(p, k)=\{p, p+1, \ldots, k\}$.

Let $m \in \mathbb{N}(1)$. In this paper we consider the difference equation of the form

$$
\begin{aligned}
& \Delta^{m} x_{n}=a_{n} f\left(n, x_{\sigma(n)}\right)+b_{n}, \\
& a_{n}, b_{n} \in \mathbb{R}, \quad f: \mathbb{N} \times \mathbb{R} \rightarrow \mathbb{R}, \quad \sigma: \mathbb{N} \rightarrow \mathbb{Z}, \quad \lim \sigma(n)=\infty .
\end{aligned}
$$

By a solution of (E) we mean a sequence $x: \mathbb{N} \rightarrow \mathbb{R}$ satisfying (E) for all large $n$. We denote the space of all sequences $x: \mathbb{N} \rightarrow \mathbb{R}$ by SQ. We denote the Banach space of all bounded sequences $x \in \mathrm{SQ}$ with the norm $\|x\|=\sup \left\{\left|x_{n}\right|: n \in \mathbb{N}\right\}$ by BS. If $x, y$ in $\mathrm{SQ}$, then $x y$ denotes the sequence defined by pointwise multiplication $x y(n)=x_{n} y_{n}$. Moreover, $|x|$ denotes the sequence defined by $|x|(n)=\left|x_{n}\right|$ for every $n$. We use the symbols 'big O' and 'small o' in the usual sense, but for $a \in \mathrm{SQ}$, we also regard $\mathrm{o}(a)$ and $\mathrm{O}(a)$ as subspaces of SQ. More precisely, let $\mathrm{o}(1)=\{x \in \mathrm{SQ}: x$ is convergent to zero $\}, \mathrm{O}(1)=\{x \in \mathrm{SQ}: x$ is bounded $\}$, and for $a \in \mathrm{SQ}$, let

$$
\mathrm{o}(a)=a \mathrm{o}(1)=\{a x: x \in \mathrm{o}(1)\}, \quad \mathrm{O}(a)=a \mathrm{O}(1)=\{a x: x \in \mathrm{O}(1)\} .
$$

For $m \in \mathbb{N}(-1)$, let $\operatorname{Pol}(m)$ denote the space $\operatorname{Ker} \Delta^{m+1}$, i.e., the space of all polynomial sequences of degree at most $m$. Now we can define asymptotically polynomial sequences.

( 2013 Migda; licensee Springer. This is an Open Access article distributed under the terms of the Creative Commons Attribution License (http://creativecommons.org/licenses/by/2.0), which permits unrestricted use, distribution, and reproduction in any medium, provided the original work is properly cited. 
We say a sequence $x \in \mathrm{SQ}$ is asymptotically polynomial of degree at most $m$ if

$$
x \in \operatorname{Pol}(m)+\mathrm{o}\left(n^{s}\right)
$$

for some $s \in(-\infty, m]$. Note that if $t, s \in \mathbb{R}$, then the condition $\mathrm{o}\left(n^{s}\right) \subset \mathrm{o}\left(n^{t}\right)$ is equivalent to $s \leq t$. Hence $\operatorname{Pol}(m)+\mathrm{o}\left(n^{s}\right) \subset \operatorname{Pol}(m)+\mathrm{o}\left(n^{t}\right)$ if $s \leq t$. In particular if $s<0$, then $\operatorname{Pol}(m)+$ $\mathrm{o}\left(n^{s}\right) \subset \operatorname{Pol}(m)+\mathrm{o}(1)$ and the inclusion is proper. Note also that if $k \in \mathbb{N}(0, m)$ and $s \in$ $(-\infty, k]$, then $x \in \operatorname{Pol}(m)+\mathrm{o}\left(n^{s}\right)$ if and only if

$$
x_{n}=c_{m} n^{m}+c_{m-1} n^{m-1}+\cdots+c_{k} n^{k}+\mathrm{o}\left(n^{s}\right)
$$

for some fixed constants $c_{m}, \ldots, c_{k}$. For $k \in \mathbb{N}(1)$, we use the factorial notation

$$
n^{(k)}=n(n-1) \cdots(n-k+1) \quad \text { with } n^{(0)}=1 .
$$

The integer part of real number $t$ is denoted by $\lfloor t\rfloor$.

The purpose of this paper is to study the asymptotic behavior of solutions of equation (E). In Section 2 we present some preliminary results. Main results appear in Sections 3 and 4 . We establish sufficient conditions under which, for some natural $k$ and for any $\varphi \in \operatorname{Pol}(m-1)$ such that $\varphi \circ \sigma=\mathrm{O}\left(n^{k}\right)$, there exists a solution $x$ of $(E)$ such that $x=\varphi+\mathrm{o}\left(n^{s}\right)$. We also give sufficient conditions under which all solutions are asymptotically polynomial. The proofs of main theorems are based on the Schauder fixed point theorem (Theorem 3.1) and on the discrete Bihari-type inequality (Theorem 4.1).

Asymptotically polynomial solutions appear in the theory of both differential and difference equations. Especially in the theory of second-order equations, the so-called asymptotically linear solutions, i.e., asymptotically polynomial solutions of degree at most one, are considered. Asymptotically linear solutions of differential equations are considered, for example, in papers [1-11]. A historical survey of this topic can be found in [12]. The asymptotic linearity of a solution $x$, called in some papers 'property (L)', usually means (passing over some additional properties of a derivative) one of the following two conditions:

$$
x(t)=a t+b+\mathrm{o}(1) \quad \text { or } \quad x(t)=a t+\mathrm{o}(t) .
$$

In [9] and [10] the condition of the form $x(t)=a t+\mathrm{o}\left(t^{d}\right)$ for some $d \in(0,1)$ is also considered.

Asymptotically polynomial solutions of differential equations of higher order appear, for example, in papers [13-17]. In [14], Naito presented necessary and sufficient conditions under which some neutral differential equation of order $m$ possesses a solution $x$ such that

$$
\lim _{t \rightarrow \infty} \frac{x(t)}{t^{k}}=a, \quad \text { for some fixed } k \in \mathbb{N}(1, m-1) \text { and } a \in \mathbb{R} .
$$

Note that the condition $\lim _{t \rightarrow \infty} x(t) / t^{k}=a$ can be written in the form $x(t)=a t^{k}+\mathrm{o}\left(t^{k}\right)$. In [17], Hasanbulli and Rogovchenko obtained sufficient conditions under which every 
nonoscillatory solution $x$ of some neutral differential equation of order $m$ has the property

$$
\lim _{t \rightarrow \infty} \frac{x(t)}{t^{m-1}}=a \in \mathbb{R}, \quad \text { i.e., } x(t)=a t^{m-1}+\mathrm{o}\left(t^{m-1}\right) .
$$

In [15], Philos, Purnaras and Tsamatos presented sufficient conditions under which, for given $m \in \mathbb{N}(1)$ and $k \in \mathbb{N}(0, m-1)$, every solution of the equation $x^{(m)}(t)=f(t, x(t))$ fulfills the condition $x(t)=c_{0}+c_{1} t+\cdots+c_{k} t^{k}+\mathrm{o}(1)$. Moreover, they obtained sufficient conditions under which, for every polynomial function $\varphi$ of degree at most $m-1$, there exists a solution $x$ of this equation such that $x(t)=\varphi(t)+\mathrm{o}(1)$.

Asymptotically linear solutions of difference equations are studied, for example, in papers [18-22]. Asymptotic linearity, similarly as in the continuous case, usually means one of the following two conditions:

$$
x_{n}=a n+b+\mathrm{o}(1) \quad \text { or } \quad x(n)=a n+\mathrm{o}(n) .
$$

Asymptotically polynomial solutions of difference equations of higher order appear, for example, in papers [23-30]. In [25], Popenda and Drozdowicz presented necessary and sufficient conditions under which the equation $\Delta^{m} x_{n}=a_{n} f\left(x_{n}\right)$ has a convergent solution (i.e., a solution that is asymptotically polynomial of degree zero). In [26], Zafer obtained sufficient conditions under which the equation $\Delta^{m} x_{n}=F\left(n, x_{g(n)}\right)+b_{n}$ has a solution $x$ such that $\lim _{n \rightarrow \infty} x_{n} / n^{(m-1)}=a \in \mathbb{R}$, i.e., $x_{n}=a n^{(m-1)}+\mathrm{o}\left(n^{(m-1)}\right)$. It is easy to see that the last condition is equivalent to $x_{n}=a n^{m-1}+\mathrm{o}\left(n^{m-1}\right)$. In [28] sufficient conditions under which, for any $\varphi \in \operatorname{Pol}(m-1)$, there exists a solution $x$ of the equation $\Delta^{m} x_{n}=a_{n} f\left(x_{n}\right)+b_{n}$ such that $x_{n}=\varphi(n)+\mathrm{o}(1)$ are presented. In [30] sufficient conditions under which every solution $x$ of the equation $\Delta^{m} x_{n}=a_{n} F\left(n, x_{g(n)}\right)+b_{n}$ has the property $x_{n}=\varphi(n)+\mathrm{o}(1)$ for some $\varphi \in \operatorname{Pol}(m-1)$ are presented. Moreover, sufficient conditions under which, for every $\varphi \in \operatorname{Pol}(m-1)$ there exists a solution $x$ of this equation such that $x_{n}=\varphi(n)+\mathrm{o}(1)$, are presented.

\section{Asymptotically polynomial sequences}

In this section we obtain some technical results which will be used in the next sections. The solutions of 'the simplest' difference equation $\Delta^{m} x_{n}=0$ are the polynomial sequences. In Theorem 2.1, which is the main result of this section, we show that if $a \in \mathrm{SQ}$ is sufficiently 'small' then the solutions of the 'equation' $\Delta^{m} x_{n}=\mathrm{O}(a)$ are asymptotically polynomial sequences. This result will be used in the proofs of our main Theorems 3.1 and 4.1. Lemmas 2.1, 2.2 and 2.3 are used in the proof of Theorem 2.1, Lemma 2.4 is used in the proof of Theorem 2.2 and Lemma 2.5 is used to justify an important example (see Remark 4.1 and Example 4.2).

Lemma 2.1 Assume that $s \in(-1, \infty), m \in \mathbb{N}(1)$ and $\Delta^{m} x_{n}=\mathrm{o}\left(n^{s}\right)$. Then $x_{n}=\mathrm{o}\left(n^{s+m}\right)$.

Proof Induction on $m$. Let $m=1$. Using de l'Hospital theorem, we obtain

$$
\begin{aligned}
\lim _{n \rightarrow \infty} \frac{\Delta n^{s+1}}{n^{s}} & =\lim _{n \rightarrow \infty} \frac{(n+1)^{s+1}-n^{s+1}}{n^{-1} n^{s+1}}=\lim _{n \rightarrow \infty} \frac{\left(1+n^{-1}\right)^{s+1}-1}{n^{-1}} \\
& =\lim _{n \rightarrow \infty} \frac{(s+1)\left(1+n^{-1}\right)^{s}\left(-n^{-2}\right)}{-n^{-2}}=s+1 .
\end{aligned}
$$


So, by the assumption $\Delta x=\mathrm{o}\left(n^{s}\right)$, we obtain

$$
\lim \frac{\Delta x_{n}}{\Delta n^{s+1}}=\lim \frac{\Delta x_{n}}{n^{s}} \frac{n^{s}}{\Delta n^{s+1}}=\lim \frac{\Delta x_{n}}{n^{s}} \lim \frac{n^{s}}{\Delta n^{s+1}}=\frac{0}{s+1}=0 .
$$

Since $s>-1$, the sequence $\left(n^{s+1}\right)$ is increasing to infinity. By the Stolz-Cesaro theorem, we obtain $x_{n}=\mathrm{o}\left(n^{s+1}\right)$. Hence the assertion is true for $m=1$. Assume that it is true for some $m \geq 1$, and let $\Delta^{m+1} x_{n}=\mathrm{o}\left(n^{s}\right)$. Then $\Delta^{m} \Delta x_{n}=\mathrm{o}\left(n^{s}\right)$, and by an inductive hypothesis, we get $\Delta x_{n}=\mathrm{o}\left(n^{s+m}\right)$. Hence, by the first part of the proof, we obtain $x_{n}=\mathrm{o}\left(n^{s+m+1}\right)$. The proof is complete.

For $k \in \mathbb{N}(0), n \in \mathbb{N}(1)$, let

$$
s_{n}^{k}=\left(\begin{array}{c}
n+k-1 \\
k
\end{array}\right)=\frac{n(n+1) \cdots(n+k-1)}{k !}=\frac{(n+k-1)^{(k)}}{k !} .
$$

Lemma 2.2 Assume that $m \in \mathbb{N}(1)$, and let the series $\sum n^{m-1} x_{n}$ be absolutely convergent. Then there exists exactly one sequence $z$ such that $z=\mathrm{o}(1)$ and $\Delta^{m} z=(-1)^{m} x$. The sequence $z$ is defined by

$$
z_{n}=\sum_{i_{1}=n}^{\infty} \sum_{i_{2}=i_{1}}^{\infty} \cdots \sum_{i_{m}=i_{m-1}}^{\infty} x_{i_{m}}=\sum_{j=0}^{\infty} s_{j+1}^{m-1} x_{n+j}=\sum_{k=n}^{\infty} s_{k-n+1}^{m-1} x_{k} .
$$

Moreover,

$$
\left|z_{n}\right| \leq \sum_{k=n}^{\infty} k^{m-1}\left|x_{k}\right| \quad \text { for any } n \in \mathbb{N}(1) .
$$

Proof The first assertion is an immediate consequence of Lemma 4 in [31]. The second assertion follows from the inequality $s_{k-n+1}^{m-1}<k^{m-1}$.

Lemma 2.3 Assume that $u$ is a positive and nondecreasing sequence, $m \in \mathbb{N}(1)$ and

$$
\sum_{n=1}^{\infty} n^{m-1} u_{n}\left|a_{n}\right|<\infty
$$

Then there exists a sequence $w \in \mathrm{o}\left(u^{-1}\right)$ such that $\Delta^{m} w=a$.

Proof Since $u$ is positive and nondecreasing, we have $\sum_{n=1}^{\infty} n^{m-1}\left|a_{n}\right|<\infty$. By Lemma 2.2, there exist sequences $x, z \in \mathrm{o}(1)$ such that $\Delta^{m} x=(-1)^{m} a$ and $\Delta^{m} z=(-1)^{m}|u a|$. Moreover, using Lemma 2.2, we obtain

$$
\begin{aligned}
\left|u_{n} x_{n}\right| & =\left|s_{1}^{m-1} u_{n} a_{n}+s_{2}^{m-1} u_{n} a_{n+1}+\cdots\right| \leq s_{1}^{m-1} u_{n}\left|a_{n}\right|+s_{2}^{m-1} u_{n}\left|a_{n+1}\right|+\cdots \\
& \leq s_{1}^{m-1} u_{n}\left|a_{n}\right|+s_{2}^{m-1} u_{n+1}\left|a_{n+1}\right|+s_{3}^{m-1} u_{n+2}\left|a_{n+2}\right|+\cdots=z_{n}=\mathrm{o}(1) .
\end{aligned}
$$

Hence $x=\mathrm{o}\left(u^{-1}\right)$, and we can take $w=(-1)^{m} x$. 
Theorem 2.1 Assume that $m \in \mathbb{N}(1), s \in(-\infty, m-1], \Delta^{m} x \in \mathrm{O}(a)$, and

$$
\sum_{n=1}^{\infty} n^{m-1-s}\left|a_{n}\right|<\infty
$$

Then $x \in \operatorname{Pol}(m-1)+\mathrm{o}\left(n^{s}\right)$.

Proof Assume that $s \leq 0$. Since $\Delta^{m} x \in \mathrm{O}(a)$, we have $\sum n^{m-1-s}\left|\Delta^{m} x_{n}\right|<\infty$. Let $u_{n}=n^{-s}$. By Lemma 2.3, there exists a sequence $w=o\left(n^{s}\right)$ such that $\Delta^{m} w=\Delta^{m} x$. Then

$$
x-w \in \operatorname{Ker} \Delta^{m}=\operatorname{Pol}(m-1) \quad \text { and } \quad x=x-w+w \in \operatorname{Pol}(m-1)+o\left(n^{s}\right) .
$$

Let $s \in(0, m-1]$. Choose $k \in \mathbb{N}(1, m-1)$ such that $k-1<s \leq k$. Then

$$
\sum_{n=1}^{\infty} n^{m-k-1} n^{k-s}\left|\Delta^{m} x\right|<\infty
$$

and by Lemma 2.3 there exists $w=\mathrm{o}\left(n^{s-k}\right)$ such that $\Delta^{m-k} w=\Delta^{m} x$. Choose $z \in \mathrm{SQ}$ such that $\Delta^{k} z=w$. Since $s-k>-1$, so by Lemma 2.1, $z=\mathrm{o}\left(n^{s}\right)$. Moreover,

$$
\Delta^{m} z=\Delta^{m-k} \Delta^{k} z=\Delta^{m-k} w=\Delta^{m} x
$$

Hence $x=x-z+z \in \operatorname{Pol}(m-1)+\mathrm{o}\left(n^{s}\right)$. The proof is complete.

Lemma 2.4 Let $s \in \mathbb{R}$ and $n \in \mathbb{N}(2)$. Then

$$
\Delta n^{s}=\sum_{k=1}^{\infty}\left(\begin{array}{l}
s \\
k
\end{array}\right) n^{s-k}
$$

Proof If $|x|<1$, then $(1+x)^{s}=\sum_{k=0}^{\infty}\left(\begin{array}{l}s \\ k\end{array}\right) x^{k}$. Hence

$$
n^{-s} \Delta n^{s}=n^{-s}\left((n+1)^{s}-n^{s}\right)=\left(1+\frac{1}{n}\right)^{s}-1=\sum_{k=0}^{\infty}\left(\begin{array}{l}
s \\
k
\end{array}\right) n^{-k}-1=\sum_{k=1}^{\infty}\left(\begin{array}{l}
s \\
k
\end{array}\right) n^{-k},
$$

and we obtain the result.

Theorem 2.2 If $m \in \mathbb{N}(1)$ and $s \in \mathbb{R}$, then

$$
\Delta^{m} n^{s}=s^{(m)} n^{s-m}+\mathrm{o}\left(n^{s-m}\right)=\mathrm{O}\left(n^{s-m}\right) .
$$

Proof First we show, by induction on $m$, that there exists a sequence $c$ such that

$$
\Delta^{m} n^{s}=s^{(m)} n^{s-m}+\sum_{k=m+1}^{\infty} c_{k} n^{s-k}
$$

For $m=1$ this assertion follows from Lemma 2.4. Assume it is true for some $m \geq 1$. By Lemma 2.1 and by an inductive hypothesis, we obtain sequences $c^{\prime}, b, b^{\prime}, b^{\prime \prime}, c^{\prime \prime}$ and $c$ such 
that

$$
\begin{aligned}
\Delta^{m+1} n^{s} & =\Delta \Delta^{m} n^{s}=\Delta\left(s^{(m)} n^{s-m}+\sum_{k=m+1}^{\infty} c_{k}^{\prime} n^{s-k}\right)=s^{(m)} \Delta n^{s-m}+\sum_{k=m+1}^{\infty} c_{k}^{\prime} \Delta n^{s-k} \\
& =s^{(m)}\left((s-m) n^{s-m-1}+\sum_{k=2}^{\infty} b_{k} n^{s-m-k}\right)+\sum_{k=m+1}^{\infty} c_{k}^{\prime}\left((s-k) n^{s-k-1}+\sum_{j=2}^{\infty} b_{j}^{\prime} n^{s-k-j}\right) \\
& =s^{(m+1)} n^{s-m-1}+\sum_{k=2}^{\infty} b_{k}^{\prime \prime} n^{s-m-k}+\sum_{k=m+1}^{\infty} c_{k}^{\prime \prime} n^{s-k-1} \\
& =s^{(m+1)} n^{s-m-1}+\sum_{k=m+2}^{\infty} b_{k-m}^{\prime \prime} n^{s-k}+\sum_{k=m+2}^{\infty} c_{k-1}^{\prime \prime} n^{s-k}=s^{(m+1)} n^{s-m-1}+\sum_{k=m+2}^{\infty} c_{k} n^{s-k} .
\end{aligned}
$$

Hence we obtain (2). The assertion (1) is an easy consequence of (2).

Lemma 2.5 Assume that $s, a \in \mathbb{R}$ and $m \in \mathbb{N}(-1)$. The following conditions are equivalent:
(1) $\left(n^{a}\right) \in \operatorname{Pol}(m)+o\left(n^{s}\right)$,
(2) $a<s$ or $a \in \mathbb{N}(0, m)$.

Proof If $a<s$, then $\left(n^{a}\right) \in \mathrm{o}\left(n^{s}\right) \subset \operatorname{Pol}(m)+\mathrm{o}\left(n^{s}\right)$. If $a \in \mathbb{N}(0, m)$, then

$$
\left(n^{a}\right) \in \operatorname{Pol}(m) \subset \operatorname{Pol}(m)+\mathrm{o}\left(n^{s}\right)
$$

Assume that $a \geq s$ and $a \notin \mathbb{N}(0, m)$. Obviously, $\left(n^{a}\right) \notin \mathrm{o}\left(n^{s}\right)$. Hence the assumption $\left(n^{a}\right) \in$ $\operatorname{Pol}(m)+\mathrm{o}\left(n^{s}\right)$ implies the existence of a nonzero constant $c$ such that $n^{a}=c n^{k}+\mathrm{o}\left(n^{k}\right)+$ $\mathrm{o}\left(n^{s}\right)$ for some $k \in \mathbb{N}(0, m)$. If $k>a$, then dividing by $n^{k}$, we obtain $\mathrm{o}(1)=c+\mathrm{o}(1)$, which is impossible. If $k<a$, then dividing by $n^{a}$, we have $1=\mathrm{o}(1)$, which is impossible too. Hence, in this case, $\left(n^{a}\right) \notin \operatorname{Pol}(m)+\mathrm{o}\left(n^{s}\right)$. The proof is complete.

\section{Asymptotically polynomial solutions}

In this section we consider the first issue of the Abstract. In Theorem 3.2 we establish sufficient conditions under which, for any $\varphi \in \operatorname{Pol}(m-1)$ and for any real $s \leq 0$, there exists a solution $x$ of $(\mathrm{E})$ such that $x=\varphi+\mathrm{o}\left(n^{s}\right)$. However, the main result of the section is more general Theorem 3.1 in which we establish sufficient conditions under which, for some natural $k$ and for any $\varphi \in \operatorname{Pol}(m-1)$ such that $\varphi \circ \sigma=\mathrm{O}\left(n^{k}\right)$, there exists a solution $x$ of (E) such that $x=\varphi+o\left(n^{s}\right)$. In the second part of the section (Theorems 3.3, 3.4 and Corollary 3.1), we present some consequences of Theorem 3.1.

Theorem 3.1 generalizes Theorem 2 of [28]. The method of the proof of Theorem 3.1 shows certain similarities to the method used in the continuous case in the proof of Theorem 1 of [15].

In this section we regard $\mathbb{N} \times \mathbb{R}$ as a metric subspace of the plane $\mathbb{R}^{2}$. Moreover, we assume $a, b \in \mathrm{SQ}, m \in \mathbb{N}(1), s \in(-\infty, 0]$ and

$$
\sum_{n=1}^{\infty} n^{m-1-s}\left|a_{n}\right|<\infty, \quad \sum_{n=1}^{\infty} n^{m-1-s}\left|b_{n}\right|<\infty .
$$


Theorem 3.1 Assume that $k \in \mathbb{N}(0), g:[0, \infty) \rightarrow[0, \infty)$ is continuous, $f$ is continuous and

$$
|f(n, t)| \leq g\left(\frac{|t|}{n^{k}}\right) \quad \text { for }(n, t) \in \mathbb{N} \times \mathbb{R}
$$

Then for any $\varphi \in \operatorname{Pol}(m-1)$ such that $\varphi \circ \sigma=\mathrm{O}\left(n^{k}\right)$, there exists a solution $x$ of $(\mathrm{E})$ such that

$$
x=\varphi+\mathrm{o}\left(n^{s}\right) .
$$

Proof Let $x$ be a solution of equation (E), and let $\varphi \in \operatorname{Pol}(m-1), \varphi(\sigma(n))=\mathrm{O}\left(n^{k}\right)$. Let us denote $y_{n}=x_{n}-\varphi(n)$. Then $\Delta^{m} y_{n}=\Delta^{m} x_{n}$ and equation (E) takes the form

$$
\Delta^{m} y_{n}=a_{n} f\left(n, y_{\sigma(n)}+\varphi_{\sigma(n)}\right)+b_{n}
$$

Hence, we have to prove that equation $\left(\mathrm{E}^{*}\right)$ has a solution $y$ such that $y=\mathrm{o}\left(n^{s}\right)$. Let $n_{0}=$ $\min \{n \in \mathbb{N}: \sigma(k) \geq 1$ for all $k \geq n\}$. For $y \in \mathrm{SQ}$, we define $\bar{y}$ and $\tilde{y}$ by

$$
\bar{y}_{n}=\left\{\begin{array}{ll}
y_{\sigma(n)}+\varphi_{\sigma(n)} & \text { for } n \geq n_{0}, \\
0 & \text { for } n<n_{0},
\end{array} \quad \tilde{y}_{n}=a_{n} f\left(n, \bar{y}_{n}\right)+b_{n} .\right.
$$

Let $T=\{y \in \mathrm{SQ}:\|y\| \leq 1\}$. Since $\varphi(\sigma(n))=\mathrm{O}\left(n^{k}\right)$, there exists a constant $L$ such that $\left(\bar{y}_{n} / n^{k}\right) \leq L$ for $y \in T$. Hence, by the continuity of $g$, there exists a constant $M \geq 1$ such that $g\left(\left|\bar{y}_{n}\right| / n^{k}\right) \leq M$ for every $y \in T$ and every $n$. Moreover,

$$
\left|\widetilde{y}_{n}\right| \leq M\left|a_{n}\right|+\left|b_{n}\right| \leq M\left(\left|a_{n}\right|+\left|b_{n}\right|\right)
$$

for every $y \in T$ and every $n$. Let

$$
d_{n}=M \sum_{j=n}^{\infty} s_{j-n+1}^{m-1}\left(\left|a_{j}\right|+\left|b_{j}\right|\right) .
$$

Choose $p$ such that $p \geq n_{0}$ and $d_{n} \leq 1$ for $n \geq p$. Let

$$
S=\left\{z \in \mathrm{SQ}:\left|z_{n}\right| \leq d_{n} \text { for } n \geq p \text { and } z_{n}=0 \text { for } n<p\right\} .
$$

Then $S \subset T$ and $S$ is a convex subset of the Banach space BS. Moreover, as in the proof of Theorem 1 in [30], one can show that $S$ is compact. If $y \in T$, then $|\widetilde{y}| \leq M(|a|+|b|)$. Hence, by (3) we have $\sum_{n=1}^{\infty} n^{m-1}\left|\tilde{y}_{n}\right|<\infty$. For $y \in T$, let

$$
H(y)(n)= \begin{cases}(-1)^{m} \sum_{j=n}^{\infty} s_{j-n+1}^{m-1} \tilde{y}_{j} & \text { for } n \geq p \\ 0 & \text { for } n<p\end{cases}
$$

Then, for $y \in S$ and $n \geq p$, by (5) and (6), we get

$$
|H(y)(n)|=\left|\sum_{j=n}^{\infty} s_{j-n+1}^{m-1} \widetilde{y}_{j}\right| \leq \sum_{j=n}^{\infty} s_{j-n+1}^{m-1}\left|\widetilde{y}_{j}\right| \leq d_{n} .
$$


Hence $H(S) \subset S$. Let $\varepsilon>0$. Choose $\varepsilon_{1}>0$ and $\varepsilon_{2}>0$ such that $\varepsilon_{1}+\varepsilon_{2}=\varepsilon$. There exists $q \geq p$ such that $2 d_{n}<\varepsilon_{2}$ for $n \geq q$. Choose $\alpha>0$ such that

$$
\alpha \sum_{j=p}^{q} s_{j-n+1}^{m-1}\left|a_{j}\right|<\varepsilon_{1} .
$$

Let $y \in S$, and let $j \in\{p, \ldots, q\}$. By the continuity of $f$ at a point $\left(j, \bar{y}_{j}\right)$, there exists $\delta_{j}>0$ such that $\left|\bar{y}_{j}-s\right|<\delta_{j}$ implies $\left|f\left(j, \bar{y}_{j}\right)-f(j, s)\right|<\alpha$. Let $\delta=\min \left\{\delta_{p}, \delta_{p+1}, \ldots, \delta_{q}\right\}$, and let $z \in S$, $\|y-z\|<\delta$. If $j \in\{p, \ldots, q\}$, then $\left|\bar{y}_{j}-\bar{z}_{j}\right|=\left|y_{\sigma(j)}-z_{\sigma(j)}\right|<\delta$. Hence

$$
\left|\widetilde{y}_{j}-\widetilde{z}_{j}\right|=\left|a_{j} f\left(j, \bar{y}_{j}\right)-a_{j} f\left(j, \bar{z}_{j}\right)\right|<\left|a_{j}\right| \alpha
$$

and

$$
\sum_{j=p}^{q} s_{j-n+1}^{m-1}\left|\widetilde{y}_{j}-\widetilde{z}_{j}\right|<\sum_{j=p}^{q} s_{j-n+1}^{m-1}\left|a_{j}\right| \alpha<\varepsilon_{1} .
$$

Moreover, by (5) and (6), we have

$$
\sum_{j=q}^{\infty} s_{j-n+1}^{m-1}\left|\widetilde{y}_{j}-\widetilde{z}_{j}\right| \leq \sum_{j=q}^{\infty} s_{j-n+1}^{m-1}\left|\widetilde{y}_{j}\right|+\sum_{j=q}^{\infty} s_{j-n+1}^{m-1}\left|\widetilde{z}_{j}\right| \leq d_{q}+d_{q}<\varepsilon_{2} .
$$

Hence

$$
\begin{aligned}
\|H(y)-H(z)\| & \left.=\sup _{n \geq p}|H(y)(n)-H(z)(n)|=\sup _{n \geq p} \mid \sum_{j=n}^{\infty} s_{j-n+1}^{m-1} \widetilde{y}_{j}-\widetilde{z}_{j}\right) \mid \\
& \leq \sum_{j=p}^{\infty} s_{j-n+1}^{m-1} \widetilde{y}_{j}-\widetilde{z}_{j}\left|\leq \sum_{j=p}^{q} s_{j-n+1}^{m-1}\right| \widetilde{y}_{j}-\widetilde{z}_{j}\left|+\sum_{j=q}^{\infty} s_{j-n+1}^{m-1}\right| \widetilde{y}_{j}-\widetilde{z}_{j} \mid<\varepsilon_{1}+\varepsilon_{2}=\varepsilon .
\end{aligned}
$$

This shows that the map $H$ is continuous. By the Schauder fixed point theorem, there exists a sequence $y \in S$ such that $H(y)=y$. Then $y_{n}=(-1)^{m} \sum_{j=n}^{\infty} s_{j-n+1}^{m-1} \tilde{y}_{j}$ for $n \geq p$. By Lemma 2.2 we obtain

$$
\Delta^{m} y_{n}=\tilde{y}_{n}=a_{n} f\left(n, \bar{y}_{n}\right)+b_{n}=a_{n} f\left(n, y_{\sigma(n)}+\varphi_{\sigma(n)}\right)+b_{n}
$$

for $n \geq p$. Hence $y$ is a solution of $\left(\mathrm{E}^{*}\right)$. Moreover, by (5) we have

$$
\left|\Delta^{m} y_{n}\right|=\left|\tilde{y}_{n}\right| \leq M\left(\left|a_{n}\right|+\left|b_{n}\right|\right) .
$$

Hence $\Delta^{m} y=\mathrm{O}(|a|+|b|)$, and by Theorem 2.1 we obtain $y \in \operatorname{Pol}(m-1)+\mathrm{o}\left(n^{s}\right)$. Moreover, $s \leq 0$ and $y=\mathrm{o}(1)$. Hence $y=\mathrm{o}\left(n^{s}\right)$. The proof is complete.

Remark 3.1 Let $k_{1}$ denote the greatest natural number such that for every polynomial $\varphi \in \operatorname{Pol}\left(k_{1}\right)$, there exists a solution $x$ of (E) such that $x=\varphi+\mathrm{o}(1)$. Note that if in Theorem 3.1 $\sigma(n)=n-p$ for some fixed integer $p$, then the condition $\varphi(\sigma(n))=\mathrm{O}\left(n^{k}\right)$ takes the form $\varphi(n)=\mathrm{O}\left(n^{k}\right)$. Hence $k_{1}=\min (k, m-1)$. But if the sequence $\sigma$ is of another form, then $k_{1}$ may be greater than $k$. In the following example, we have $k=1$ and $k_{1}=2$. 
Example 3.1 Let $m=3, s=0, k=1, \sigma(n)=\lfloor\sqrt{n}\rfloor, b_{n}=0, g(x)=x$,

$$
f(n, x)=\frac{x}{n}, \quad a_{n}=-\frac{6 n \sigma(n)}{(\sigma(n)+1)(n+3)^{(3)}} .
$$

Then the conditions of Theorem 3.1 are satisfied and equation (E) takes the form

$$
\Delta^{3} x_{n}=-\frac{6\lfloor\sqrt{n}\rfloor}{(\lfloor\sqrt{n}\rfloor+1)(n+3)^{(3)}} x_{\lfloor\sqrt{n}\rfloor} .
$$

Note, that for a polynomial $\varphi$, the condition $\varphi(\lfloor\sqrt{n}\rfloor)=\mathrm{O}(n)$ is equivalent to the condition $\varphi \in \operatorname{Pol}(2)$. Thus, by Theorem 3.1, for any $\varphi \in \operatorname{Pol}(2)$, equation (7) has a solution $\left(x_{n}\right)$ such that $x_{n}=\varphi_{n}+\mathrm{o}(1)$. One such solution is $x_{n}=n^{2}+\frac{1}{n}$.

Theorem 3.2 Assume that $\sigma(n)=\mathrm{O}(n), g:[0, \infty) \rightarrow[0, \infty)$ is continuous, $f$ is continuous and

$$
|f(n, t)| \leq g\left(\frac{|t|}{n^{m-1}}\right) \quad \text { for }(n, t) \in \mathbb{N} \times \mathbb{R} .
$$

Then, for any $\varphi \in \operatorname{Pol}(m-1)$, there exists a solution $x$ of $(\mathrm{E})$ such that $x=\varphi+\mathrm{o}\left(n^{s}\right)$.

Proof Let $\varphi \in \operatorname{Pol}(m-1)$. Choose $M, K$ such that $\left|\varphi(n) / n^{m-1}\right| \leq M$ and $\sigma(n) / n \leq K$. Then

$$
\left|\frac{\varphi(\sigma(n))}{n^{m-1}}\right|=\left|\frac{\varphi(\sigma(n)) \sigma(n)^{m-1}}{\sigma(n)^{m-1} n^{m-1}}\right| \leq M K^{m-1}
$$

Hence $\varphi \circ \sigma=\mathrm{O}\left(n^{m-1}\right)$. Now the assertion follows from Theorem 3.1.

We say that a function $f: \mathbb{N} \times \mathbb{R} \rightarrow \mathbb{R}$ is locally equibounded if for every $t \in \mathbb{R}$, there exists a neighborhood $U$ of $t$ such that $f$ is bounded on $\mathbb{N} \times U$. Obviously, every bounded function $f: \mathbb{N} \times \mathbb{R} \rightarrow \mathbb{R}$ is locally equibounded.

Example 3.2 Let $f_{1}(n, t)=t$ and $f_{2}(n, t)=n$. Then $f_{1}$ is continuous, unbounded and locally equibounded, $f_{2}$ is continuous but not locally equibounded.

Example 3.3 Let $g: \mathbb{R} \rightarrow \mathbb{R}$ be continuous, and let $f(n, t)=g(t)$. Then $f$ is continuous and locally equibounded. If $g_{0}, \ldots, g_{p-1}: \mathbb{R} \rightarrow \mathbb{R}$ are continuous and $f(n, t)=g_{n \bmod p}(t)$, then $f$ is continuous and locally equibounded.

Example 3.4 Assume that $g, h: \mathbb{R} \rightarrow \mathbb{R}$ are continuous, $\alpha, \beta \in \mathrm{SQ}$ are bounded, and let $f(n, t)=\alpha_{n} g(t)+\beta_{n} h(t)$. Then $f$ is continuous and locally equibounded.

Theorem 3.3 If $c \in \mathbb{R}, U$ is a neighborhood of $c$ and the function $f \mid \mathbb{N} \times U$ is bounded and continuous, then there exists a solution $x$ of $(\mathrm{E})$ such that $x=c+\mathrm{o}\left(n^{s}\right)$. Moreover, if $f$ is continuous and locally equibounded, then for any $c \in \mathbb{R}$, there exists a solution $x$ of $(\mathrm{E})$ such that $x=c+\mathrm{o}\left(n^{s}\right)$. 
Proof Choose $\alpha, \beta \in \mathbb{R}$ such that $\alpha<c<\beta$ and $[\alpha, \beta] \subset U$. Let

$$
f_{1}: \mathbb{N} \times \mathbb{R} \rightarrow \mathbb{R}, \quad f_{1}(n, t)= \begin{cases}f(n, \alpha) & \text { for } t \leq \alpha, \\ f(n, t) & \text { for } t \in(\alpha, \beta), \\ f(n, \beta) & \text { for } t \geq \beta\end{cases}
$$

Then $f_{1}$ is continuous and bounded. Choose $M>0$ such that $\left|f_{1}(n, t)\right| \leq M$ for any $(n, t)$. Let $g:[0, \infty) \rightarrow[0, \infty), g(t)=M$, and let $k \geq m-1$. Then

$$
\left|f_{1}(n, t)\right| \leq M=g\left(|t| / n^{k}\right)
$$

for any $(n, t) \in \mathbb{N} \times \mathbb{R}$. Hence, by Theorem 3.1, there exists a solution $x$ of the equation

$$
\Delta^{m} x_{n}=a_{n} f_{1}\left(n, x_{\sigma(n)}\right)+b_{n}
$$

such that $x=c+o\left(n^{s}\right)$. Since $\sigma(n) \rightarrow \infty, x_{\sigma(n)} \in(\alpha, \beta)$ for large $n$. Hence $f_{1}\left(n, x_{\sigma(n)}\right)=$ $f\left(n, x_{\sigma(n)}\right)$ for large $n$. Therefore $x$ is a solution of (E). The second assertion is an easy consequence of the first one.

Theorem 3.4 Assume that $\sigma(n)=\mathrm{O}\left(n^{p}\right)$ for some $p>0, \lambda \in(0, \infty), \varphi \in \operatorname{Pol}(m-1)$ and that at least one of the following conditions is satisfied:

(1) $f$ is continuous and bounded on $\mathbb{N} \times[\lambda, \infty)$ and $\varphi(n) \rightarrow \infty$,

(2) $f$ is continuous and bounded on $\mathbb{N} \times(-\infty, \lambda]$ and $\varphi(n) \rightarrow-\infty$,

(3) $f$ is continuous and bounded on $\mathbb{N} \times((-\infty,-\lambda] \cup[\lambda, \infty))$ and $\varphi$ is nonconstant.

Then there exists a solution $x$ of $(\mathrm{E})$ such that $x=\varphi+\mathrm{o}\left(n^{s}\right)$.

Proof Assume (1) is satisfied. Let

$$
f_{1}: \mathbb{N} \times \mathbb{R} \rightarrow \mathbb{R}, \quad f_{1}(n, t)= \begin{cases}f(n, \lambda) & \text { for } t \leq \lambda \\ f(n, t) & \text { for } t>\lambda\end{cases}
$$

Then $f_{1}$ is continuous and bounded. Choose $M>0$ such that $\left|f_{1}(n, t)\right| \leq M$ for any $(n, t)$. Let $g:[0, \infty) \rightarrow[0, \infty), g(t)=M$, and let $k \geq(m-1) p$. Then $\varphi \circ \sigma=\mathrm{O}\left(n^{k}\right)$ and

$$
\left|f_{1}(n, t)\right| \leq M=g\left(|t| / n^{k}\right)
$$

for any $(n, t) \in \mathbb{N} \times \mathbb{R}$. Then, by Theorem 3.1, there exists a solution $x$ of the equation

$$
\Delta^{m} x_{n}=a_{n} f_{1}\left(n, x_{\sigma(n)}\right)+b_{n}
$$

such that $x=\varphi+\mathrm{o}\left(n^{s}\right)$. Moreover, $x_{\sigma(n)}>\lambda$ for large $n$. Therefore $x$ is a solution of (E). The assertion (1) is proved. (2) is analogous to (1) and (3) is a consequence of (1) and (2).

Corollary 3.1 Assume that $\sigma(n)=\mathrm{O}\left(n^{p}\right)$ for some $p>0, c \in \mathbb{R}, \lambda \in(0, \infty), \varphi \in \operatorname{Pol}(m-1)$, $g: \mathbb{R} \rightarrow \mathbb{R}$ and that at least one of the following conditions is satisfied:

(1) $g$ is continuous on some neighborhood of $c$ and $\varphi=c$,

(2) $g$ is continuous and bounded on $[\lambda, \infty)$ and $\varphi(n) \rightarrow \infty$, 
(3) $g$ is continuous and bounded on $(-\infty, \lambda]$ and $\varphi(n) \rightarrow-\infty$,

(4) $g$ is continuous and bounded on $(-\infty,-\lambda] \cup[\lambda, \infty)$ and $\varphi$ is nonconstant.

Then there exists a solution $x$ of the equation

$$
\Delta^{m} x_{n}=a_{n} g\left(x_{\sigma(n)}\right)+b_{n}
$$

such that $x=\varphi+\mathrm{o}\left(n^{s}\right)$.

Proof This is a consequence of Theorems 3.3 and 3.4.

Example 3.5 Assume that $(-1)^{m} a_{n}>0, b_{n}=0, s=0, c \in \mathbb{R}$ and

$$
g(t)= \begin{cases}1 & \text { for } t \leq c \\ 0 & \text { for } t>c\end{cases}
$$

By Corollary 3.1, for every nonconstant polynomial $\varphi \in \operatorname{Pol}(m-1)$, there exists a solution $x$ of (E1) such that $x=\varphi+\mathrm{o}(1)$. Moreover, by Corollary 3.1, for every real $c_{1} \neq c$, there exists a solution $x$ of (E1) such that $x=c_{1}+\mathrm{o}(1)$. We will show that a solution of (E1), which is convergent to $c$, does not exist. Assume that $m$ is even. Then $a_{n}>0$. Let $x$ be a solution of (E1) such that $\lim x_{n}=c$. Then

$$
\Delta \Delta^{m-1} x_{n}=\Delta^{m} x=a_{n} g\left(x_{\sigma(n)}\right) \geq 0
$$

for large $n$. Moreover, $\Delta^{m-1} x_{n}=\Delta^{m-1}(c+o(1))=o(1)$. Hence $\Delta^{m-1} x_{n} \leq 0$ for large $n$. Thus $\Delta \Delta^{m-2} x_{n} \leq 0$ for large $n$ and so on. After $(m-1)$-steps, we obtain $\Delta x_{n} \leq 0$ for large $n$. Choose $p$ such that $\Delta x_{n} \leq 0$ for $n \geq p$. Then $x_{n} \geq c$ for $n \geq p$. If $x_{m}=c$ for some $m \geq p$, then $x_{n}=c$ for every $n \geq m$ and $\Delta^{m} x_{n}=0$ for $n \geq m$. On the other hand, if $\sigma(n) \geq m$, we obtain $\Delta^{m} x_{n}=a_{n} g\left(x_{\sigma(n)}\right)=a_{n} g(c)=a_{n}>0$, which is impossible. Hence $x_{n}>c$ for $n \geq p$. Choose $p_{1}$ such that $\sigma(n) \geq p$ for every $n \geq p_{1}$. Then $g\left(x_{\sigma(n)}\right)=0$ for $n \geq p_{1}$. Hence $\Delta^{m} x_{n}=0$ for $n \geq p_{1}$, and there exists a polynomial sequence $\varphi \in \operatorname{Pol}(m-1)$ such that $x_{n}=\varphi(n)$ for $n \geq p_{1}$. But the only polynomial $\varphi \in \operatorname{Pol}(m-1)$ which satisfies the condition $\varphi(n)=c+\mathrm{o}(1)$ is the constant polynomial $\varphi(n)=c$. Hence we again obtain $x_{n}=c$ for large $n$, which is impossible. Similarly, if $m$ is odd, one can show that a solution of (E1), which is convergent to $c$, does not exist.

\section{Approximations of solutions}

In this section we consider the second issue of the Abstract. In Theorem 4.1 we establish sufficient conditions under which, for given real $s \leq m-1$, all solutions $x$ of (E) satisfy the condition $x=\varphi+\mathrm{o}\left(n^{s}\right)$ for some $\varphi \in \operatorname{Pol}(m-1)$. In the second part of the section, we present some consequences of Theorem 4.1. Moreover, in Example 4.2 we show that the assertion of Theorem 4.1 is in some sense optimal.

Theorem 4.1 generalizes Theorem 4 of [26]. The way Theorem 4.1 is proved partially resembles the methods used in the continuous case in the proofs of Theorem 1 in [5] and Theorem 2 in [15].

In this section we assume $a, b \in \mathrm{SQ}, m \in \mathbb{N}(1), s \in(-\infty, m-1]$ and

$$
\sum_{n=1}^{\infty} n^{m-1-s}\left|a_{n}\right|<\infty, \quad \sum_{n=1}^{\infty} n^{m-1-s}\left|b_{n}\right|<\infty .
$$


Lemma 4.1 Assume that $\varphi:[0, \infty) \rightarrow[0, \infty)$ is a continuous and nondecreasing function such that $\varphi(t)>0$ for $t \geq 1$ and $\int_{1}^{\infty} \varphi(s)^{-1} d s=\infty$. Let $c>0$ and let $a, u$ be sequences of nonnegative real numbers such that

$$
\sum_{n=1}^{\infty} a_{n}<\infty \text { and } u_{n} \leq c+\sum_{i=1}^{n-1} a_{n} \varphi\left(u_{n}\right) \text { for any } n .
$$

Then the sequence $u$ is bounded.

Proof The assertion is an easy consequence of Theorem 1 in [32].

The next lemma can be found in [33].

Lemma 4.2 Let $\left(a_{n}\right)$ be a sequence of real numbers, and let $p, m \in \mathbb{N}(1), n \in \mathbb{N}(p+1)$. Then

$$
\sum_{i_{m}=p}^{n-1} \sum_{i_{m-1}=p}^{i_{m}-1} \cdots \sum_{i_{1}=p}^{i_{2}-1} a_{i_{1}}=\sum_{k=p}^{n-1} \frac{(n-k-1)^{\frac{m-1}{}}}{(m-1) !} a_{k} .
$$

Theorem 4.1 Assume that $\sigma(n) \leq n, g:[0, \infty) \rightarrow[0, \infty)$ is nondecreasing and continuous, $f$ is continuous, and assume that

$$
|f(n, t)| \leq g\left(\frac{|t|}{n^{m-1}}\right) \quad \text { for all }(n, t) \in \mathbb{N} \times \mathbb{R}, \quad \int_{1}^{\infty} \frac{d t}{g(t)}=\infty
$$

and $x$ is a solution of $(\mathrm{E})$. Then $x \in \operatorname{Pol}(m-1)+\mathrm{o}\left(n^{s}\right)$.

Proof Assume that $x$ is a solution of (E). Then, by (9) for large $n$, we have

$$
\left|\Delta^{m} x_{n}\right| \leq\left|a_{n}\right| g\left(\frac{\left|x_{\sigma(n)}\right|}{n^{m-1}}\right)+\left|b_{n}\right|
$$

From the identity $x_{n}=x_{1}+\sum_{i=1}^{n-1} \Delta x_{i}$, we get $\left|x_{n}\right| \leq\left|x_{1}\right|+\sum_{i=1}^{n-1}\left|\Delta x_{i}\right|$. Similarly, $\left|\Delta x_{i}\right| \leq$ $\left|\Delta x_{1}\right|+\sum_{j=1}^{i-1}\left|\Delta^{2} x_{j}\right|$. Hence we have

$$
\left|x_{n}\right| \leq\left|x_{1}\right|+\sum_{i=1}^{n-1}\left(\left|\Delta x_{1}\right|+\sum_{j=1}^{i-1}\left|\Delta^{2} x_{j}\right|\right) \leq \sum_{i=1}^{n-1}\left(\left|x_{1}\right|+\left|\Delta x_{1}\right|+\sum_{j=1}^{i-1}\left|\Delta^{2} x_{j}\right|\right) .
$$

Analogously, $\left|\Delta^{2} x_{j}\right| \leq\left|\Delta^{2} x_{1}\right|+\sum_{k=1}^{j-1}\left|\Delta^{3} x_{k}\right|$ and then

$$
\begin{aligned}
\left|x_{n}\right| & \leq \sum_{i=1}^{n-1}\left(\left|x_{1}\right|+\left|\Delta x_{1}\right|+\sum_{j=1}^{i-1}\left(\left|\Delta^{2} x_{1}\right|+\sum_{k=1}^{j-1}\left|\Delta^{3} x_{k}\right|\right)\right) \\
& \leq \sum_{i=1}^{n-1} \sum_{j=1}^{i-1}\left(\left|x_{1}\right|+\left|\Delta x_{1}\right|+\left|\Delta^{2} x_{1}\right|+\sum_{k=1}^{j-1}\left|\Delta^{3} x_{k}\right|\right),
\end{aligned}
$$

and so on. After $m-1$ steps, we get

$$
\left|x_{n}\right| \leq \sum_{i_{m}=1}^{n-1} \sum_{i_{m-1}=1}^{i_{m}-1} \cdots \sum_{i_{2}=1}^{i_{3}-1}\left(\left|x_{1}\right|+\left|\Delta x_{1}\right|+\cdots+\left|\Delta^{m} x_{1}\right|+\sum_{i_{1}=1}^{i_{2}-1}\left|\Delta^{m} x_{i_{1}}\right|\right) \text {. }
$$


Let $A=\left|x_{1}\right|+\left|\Delta x_{1}\right|+\cdots+\left|\Delta^{m} x_{1}\right|$. By Lemma 4.2 we obtain

$$
\left|x_{n}\right| \leq \sum_{k=1}^{n-1} \frac{(n-k-1)^{\frac{m-2}{2}}}{(m-2) !}\left(A+\sum_{j=1}^{n-1}\left|\Delta^{m} x_{j}\right|\right) \text {. }
$$

Using the inequality

$$
\frac{(n-k-1)^{\frac{m-2}{}}}{(m-2) !} \leq n^{m-2}
$$

we have $\left|x_{n}\right| \leq n^{m-1}\left(A+\sum_{j=1}^{n-1}\left|\Delta^{m} x_{j}\right|\right)$. Hence

$$
\begin{aligned}
\frac{\left|x_{\sigma(n)}\right|}{n^{m-1}} & \leq \frac{\left|x_{\sigma(n)}\right|}{\sigma(n)^{m-1}} \leq A+\sum_{j=1}^{\sigma(n)-1}\left|\Delta^{m} x_{j}\right| \leq A+\sum_{j=1}^{n-1}\left|\Delta^{m} x_{j}\right| \\
& \leq A+\sum_{j=1}^{n-1}\left(\left|a_{j}\right| g\left(\frac{\left|x_{\sigma(j)}\right|}{j^{m-1}}\right)+\left|b_{j}\right|\right) \leq B+\sum_{j=1}^{n-1}\left|a_{j}\right| g\left(\frac{\left|x_{\sigma(j)}\right|}{j^{m-1}}\right)
\end{aligned}
$$

where $B=A+\sum_{j=1}^{\infty}\left|b_{j}\right|$. By Lemma 4.1, the sequence $\left(\left|x_{\sigma(n)}\right| / n^{m-1}\right)$ is bounded. Hence, by (10) and the continuity of $g$, there exists a constant $M \geq 1$ such that

$$
\left|\Delta^{m} x_{n}\right| \leq\left|a_{n}\right| M+\left|b_{n}\right| \leq M\left(\left|a_{n}\right|+\left|b_{n}\right|\right)
$$

for all large $n$. Hence $\Delta^{m} x=\mathrm{O}(|a|+|b|)$, and from Theorem 2.1, we obtain $x \in \operatorname{Pol}(m-1)+$ $\mathrm{o}\left(n^{s}\right)$. The proof is complete.

Corollary 4.1 Assume that $\sigma(n) \leq n, \alpha \in(0,1], p \geq \alpha(m-1)$ and $x$ is a solution of the equation

$$
\Delta^{m} x_{n}=a_{n} \frac{\left|x_{\sigma(n)}\right|^{\alpha}}{n^{p}}+b_{n}
$$

Then $x \in \operatorname{Pol}(m-1)+\mathrm{o}\left(n^{s}\right)$.

Proof Let $g:[0, \infty) \rightarrow[0, \infty), g(t)=t^{\alpha}$ and $f(n, t)=|t|^{\alpha} / n^{p}$. Then

$$
|f(n, t)|=f(n, t)=\frac{|t|^{\alpha}}{n^{p}} \leq \frac{|t|^{\alpha}}{n^{\alpha(m-1)}}=\left(\frac{|t|}{n^{m-1}}\right)^{\alpha}=g\left(\frac{|t|}{n^{m-1}}\right) .
$$

Hence the assertion follows from Theorem 4.1.

Corollary 4.2 Assume that $\sigma(n) \leq n, \alpha \in(0,1], p \geq \alpha(m-1)$,

$$
\sum_{n=1}^{\infty} n^{m-1-s+p}\left|a_{n}\right|<\infty, \quad \sum_{n=1}^{\infty} n^{m-1-s}\left|b_{n}\right|<\infty
$$

and $x$ is a solution of the equation $\Delta^{m} x_{n}=a_{n}\left|x_{\sigma(n)}\right|^{\alpha}+b_{n}$. Then $x \in \operatorname{Pol}(m-1)+\mathrm{o}\left(n^{s}\right)$. 
Proof If $a_{n}^{\prime}=n^{p} a_{n}$, then $\sum_{n=1}^{\infty} n^{m-1-s}\left|a_{n}^{\prime}\right|<\infty$ and $x$ is a solution of the equation

$$
\Delta^{m} x_{n}=a_{n}^{\prime} \frac{\left|x_{\sigma(n)}\right|^{\alpha}}{n^{p}}+b_{n}
$$

Hence $x \in \operatorname{Pol}(m-1)+\mathrm{o}\left(n^{s}\right)$ by Corollary 4.1 .

Example 4.1 Let $m \geq 2, s=1$,

$$
f(n, x)=0, \quad g(x)=1, \quad a_{n}=0, \quad b_{n}=(-1)^{m-1} \frac{(m-1) !}{(n+m)^{\underline{m}}} .
$$

Then the conditions of Theorem 4.1 are satisfied and equation (E) takes the form

$$
\Delta^{m} x_{n}=(-1)^{m-1} \frac{(m-1) !}{(n+m)^{\underline{m}}}
$$

The general solution $x$ of (11) can be written in the form

$$
x_{n}=c_{m-1} n^{m-1}+c_{m-2} n^{m-2}+\cdots+c_{1} n+c_{0}+\sum_{k=1}^{n-1} \frac{1}{k} \text {. }
$$

Using the formula $\sum_{k=1}^{n-1} k^{-1}=\ln n+\mathrm{o}(1)=\mathrm{o}(n)$, we obtain $x \in \operatorname{Pol}(m-1)+\mathrm{o}(n)$. Note that $x \notin \operatorname{Pol}(m-1)+\mathrm{o}(1)$.

Remark 4.1 If the assumptions of Theorem 4.1 are satisfied, then every solution of (E) is an element of the space

$$
\operatorname{Pol}(m-1)+o\left(n^{s}\right)
$$

We will show that for every $\varepsilon>0$, there exist sequences $a, b$ and a function $f$ such that the assumptions of Theorem 4.1 are satisfied and equation (E) has a solution $x$ such that

$$
x \notin \operatorname{Pol}(m-1)+\mathrm{o}\left(n^{s-\varepsilon}\right) .
$$

Example 4.2 Let $m \in \mathbb{N}(1), \sigma(n)=n, \varepsilon>0,0<\lambda<\varepsilon, s \in(-\infty, m-1], s-\lambda \notin \mathbb{N}(0), b_{n}=0$, $\beta=s-\lambda, \mu=m-s-1+\lambda, 0<\alpha<\lambda / \mu, x_{n}=n^{\beta}$,

$$
g(t)=t^{\alpha}, \quad f(n, t)=g\left(\frac{|t|}{n^{m-1}}\right)=\left(\frac{|t|}{n^{m-1}}\right)^{\alpha} \quad \text { and } \quad a_{n}=n^{\alpha \mu} \Delta^{m} n^{\beta} .
$$

Since $0<\alpha<1$, we have $\int_{1}^{\infty} g^{-1}(t) d t=\infty$. By Lemma 2.5,

$$
x \in \operatorname{Pol}(m-1)+\mathrm{o}\left(n^{s}\right) \text { and } x \notin \operatorname{Pol}(m-1)+\mathrm{o}\left(n^{s-\varepsilon}\right) .
$$

By Theorem 2.2,

$$
n^{m-s-1} a_{n}=n^{m-s-1} n^{\alpha \mu} \Delta^{m} n^{\beta}=n^{m-s-1+\alpha \mu} \mathrm{O}\left(n^{\beta-m}\right)=\mathrm{O}\left(n^{\alpha \mu+\beta-s-1}\right) .
$$


Moreover, $\alpha \mu+\beta-s-1<\lambda+\beta-s-1=-1$. Hence $n^{m-s-1} a_{n}=\mathrm{O}\left(n^{\delta}\right)$ for some $\delta<-1$. Therefore $\sum_{n=1}^{\infty} n^{m-s-1}\left|a_{n}\right|<\infty$. Moreover,

$$
a_{n} f\left(n, x_{n}\right)+b_{n}=n^{\alpha \mu} \Delta^{m} n^{\beta}\left(n^{1-m} n^{\beta}\right)^{\alpha}=n^{\alpha(\mu+1-m+\beta)} \Delta^{m} n^{\beta}=n^{0} \Delta^{m} n^{\beta}=\Delta^{m} x_{n} .
$$

Hence $x$ is a solution of the equation $\Delta^{m} x_{n}=a_{n} f\left(n, x_{n}\right)+b_{n}$.

\section{Competing interests}

The author declares that he has no competing interests.

\section{Acknowledgements}

The author would like to thank the referees for their valuable suggestions and comments.

Received: 19 October 2012 Accepted: 11 March 2013 Published: 4 April 2013

\section{References}

1. Hallam, TG: Asymptotic integration of second order differential equation with integrable coefficients. SIAM J. Appl. Math. 19, 430-439 (1970)

2. Rogovchenko, YV, Villari, G: Asymptotic behaviour of solutions for second order nonlinear autonomous differential equations. Nonlinear Differential Equations Appl. 4, 271-282 (1997)

3. Rogovchenko, YV: On the asymptotic behavior of solutions for a class of second order nonlinear differential equations. Collect. Math. 49, 113-120 (1998)

4. Rogovchenko, SP, Rogovchenko, YV: Asymptotic behavior of solutions of second order nonlinear differential equations. Portugal. Math. 57, 17-33 (2000)

5. Dzurina, J: Asymptotic behavior of solutions of neutral nonlinear differential equations. Arch. Math. (Brno) 38(4), 319-325 (2002)

6. Mustafa, OG, Rogovchenko, YV: Global existence of solutions with prescribed asymptotic behavior for second-order nonlinear differential equations. Nonlinear Anal. 51, 339-368 (2002)

7. Lipovan, $\mathrm{O}$ : On the asymptotic behavior of the solutions to a class of second order nonlinear differential equations. Glasgow Math. J. 45, 179-187 (2003)

8. Mustafa, OG, Rogovchenko, YV: Global existence and asymptotic behavior of solutions of nonlinear differential equations. Funkcial. Ekvac. 47, 167-186 (2004)

9. Mustafa, OG, Rogovchenko, YV: Asymptotic integration of a class of nonlinear differential equations. Appl. Math. Lett. $19,849-853(2006)$

10. Mustafa, OG, Rogovchenko, YV: Positive solutions of second-order differential equations with prescribed behavior of the first derivative. In: Agarwal, RP, Perera, K (eds.) Proc. Conf. Differential and Difference Eqns. Appl., Melbourne, Florida, August 1-5, 2005, pp. 835-842. Hindawi Publ. Corp., New York (2007)

11. Mustafa, OG, Tunc, C: Asymptotically linear solutions of differential equations via Lyapunov functions. Appl. Math. Comput. 215(8), 3076-3081 (2009)

12. Agarwal, RP, Djebali, S, Moussaoui, T, Mustafa, OG: On the asymptotic integration of nonlinear differential equations J. Comput. Appl. Math. 202, 352-376 (2007)

13. Kusano, T, Trench, WF: Global existence theorems for solutions of nonlinear differential equations with prescribed asymptotic behavior. J. London Math. Soc. 31, 478-486 (1985)

14. Naito, M: An asymptotic theorem for a class of nonlinear neutral differential equations. Czechoslovak Math. J. 48, 419-432 (1998)

15. Philos, CG, Purnaras, IK, Tsamatos, PC: Asymptotic to polynomials solutions for nonlinear differential equations. Nonlinear Anal. 59, 1157-1179 (2004)

16. Philos, CG, Tsamatos, PC: Solutions approaching polynomials at infinity to nonlinear ordinary differential equations Electron. J. Differential Equations 79, 1-25 (2005)

17. Hasanbulli, M, Rogovchenko, YV: Asymptotic behavior nonoscillatory solutions to $n$-th order nonlinear neutral differential equations. Nonlinear Anal. 69, 1208-1218 (2008)

18. Drozdowicz, A, Popenda, J: Asymptotic behavior of the solutions of the second order difference equations. Proc. Am. Math. Soc. 99(1), 135-140 (1987)

19. Li, WT, Cheng, SS: Asymptotically linear solutions of a discrete Emden-Fowler equation. Far East J. Math. Sci. 6(4), 521-542 (1998)

20. Migda, M, Migda, J: Asymptotic properties of the solutions of the second order difference equations. Arch. Math. (Brno) 34, 467-476 (1998)

21. Drozdowicz, A, Migda, J: On asymptotic behavior of solutions of some difference equation. Math. Slovaca 52(2), 207-214 (2002)

22. Migda, M, Migda, J: Asymptotic properties of solutions of second-order neutral difference equations. Nonlinear Anal. 63, 789-799 (2005)

23. Popenda, J, Werbowski, J: On the asymptotic behaviour of the solutions of difference equations of second order. Comment. Math. Prace Mat. 22, 135-142 (1980)

24. Popenda, J: Asymptotic properties of solutions of difference equations. Proc. Indian Acad. Sci. Math. Sci. 95(2), 141-153 (1986)

25. Drozdowicz, A, Popenda, J: Asymptotic behavior of the solutions of an $n$-th order difference equations. Annales Soc. Math. Pol., Com. Math. XXIX, 161-168 (1990) 
26. Zafer, A: Oscillatory and asymptotic behavior of higher order difference equations. Math. Comput. Modelling 21(4), 43-50 (1995)

27. Gleska, A, Werbowski, J: Comparison theorems for the asymptotic behavior of solutions of nonlinear difference equations. J. Math. Anal. Appl. 226(2), 456-465 (1998)

28. Migda, M, Migda, J: On the asymptotic behavior of solutions of higher order nonlinear difference equations. Nonlinear Anal. 47(7), 4687-4695 (2001)

29. Wang, Z, Sun, J: Asymptotic behavior of solutions of nonlinear higher-order neutral type difference equations. J. Difference Equ. Appl. 12, 419-432 (2006)

30. Migda, J: Asymptotic properties of solutions of nonautonomous difference equations. Arch. Math. (Brno) 46, 1-11 (2010)

31. Migda, J: Asymptotic properties of solutions of higher order difference equations. Math. Bohem. 135(1), $29-39$ (2010)

32. Demidovič, VB: A certain criterion for the stability of difference equations. (Russian). Differ. Uravn. (Minsk) 5 , 1247-1255 (1969)

33. Migda, M: On unstable neutral difference equations. Indian J. Pure and Appl. Math. 36(10), 557-567 (2005)

doi:10.1186/1687-1847-2013-92

Cite this article as: Migda: Asymptotically polynomial solutions of difference equations. Advances in Difference Equations 2013 2013:92

\section{Submit your manuscript to a SpringerOpen ${ }^{\circ}$ journal and benefit from:}

- Convenient online submission

- Rigorous peer review

- Immediate publication on acceptance

- Open access: articles freely available online

- High visibility within the field

- Retaining the copyright to your article 